\title{
VARIACIÓN DE LA MASA CORPORAL GRASA POR ANTROPOMETRÍA Y BIOIMPEDANCIA EN ESCOLARES JUJEÑOS
}

\section{BODY FAT VARIATION BY ANTHROPOMETRY AND BIOIMPEDANCE IN JUJENEAN SCHOOL CHILDREN}

\author{
Jorge Ivan Martinez ${ }^{1 *}$, José Edgardo Dipierri1,2, Ignacio Bejarano3 ${ }^{3}$ Yolanda Quispe ${ }^{3}$ y Emma \\ Alfaro ${ }^{1,2}$ \\ ${ }^{I}$ INECOA-CONICET- Universidad Nacional de Jujuy. San Salvador de Jujuy. Jujuy. Argentina \\ ${ }^{2}$ Instituto de Biología de la Altura. Universidad Nacional de Jujuy. San Salvador de Jujuy. Jujuy. Argentina \\ ${ }^{3}$ Unidad de Investigación Antropología Biológica (UIAB). Facultad de Humanidades y Ciencias Sociales. Universidad Nacional de \\ Jujuy. San Salvador de Jujuy. Jujuy. Argentina
}

PALABRAS CLAVE impedancia bioeléctrica; pliegues cutáneos; Índice de masa corporal; Jujuy

\begin{abstract}
RESUMEN El objetivo del presente artículo es comparar distintos métodos de cálculo del porcentaje de grasa corporal (\%GC) por antropometría y bioimpedancia (BIA) en una población infanto-juvenil jujeña y analizar la variación de la masa grasa (MG), masa magra (MM), y de los índices de masa grasa (IMG) y masa magra (IMM). Se realizó un estudio transversal en 232 escolares de 12 a 17 años de la ciudad de San Salvador de Jujuy. Se relevaron datos de talla, peso y pliegues tricipital y subescapular. Se determinó el Índice de Masa corporal (IMC) y el \%GC por BIA (Maltron BF-900) y antropometría (ecuaciones de Deurenberg y Slaugther). Se calculó media y desvío estándar y las diferencias por sexo y edad se establecieron mediante análisis de la varianza. Se realizó una correlación de Pearson entre los distintos mé-
\end{abstract}

todos utilizados para estimar el \%GC y la concordancia se analizó con Bland-Altman. Las mujeres presentaron valores superiores de IMC y pliegues bicipital y tricipital y mayores porcentajes de sobrepeso y obesidad. Los \%GC más altos se obtuvieron con BIA en mujeres y con Slaugther en varones. Las mujeres registraron valores promedio significativamente más altos del IMG que los varones con los 3 métodos. La correlación más alta del \%GC se observó entre BIA y Deurenberg, seguida por Slaugther y Deurenberg; sin embargo, no se observó concordancia entre los 3 métodos. Dada la accesibilidad de la estimación del \%GC con la ecuación de Deurenberg se propone la aplicación de esta metodología en estudios epidemiológicos de obesidad. Rev Arg Antrop Biol 20(1), 2018. doi:10.17139/raab.2018.0020.01.05

\section{KEY WORDS bioelectrical impedance; skinfold thickness; body mass index; Jujuy}

ABSTRACT The objective of the present study is to compare different methods of calculating body fat percentage (BF\%) by anthropometry and bioimpedance (BIA) in an infant-juvenile population, and to analyze the variation of fat mass (FM), fat-free mass (FFM), and FMI and FFMI index. A cross-sectional study was carried out on 232 schoolchildren aged 12-17 years in the city of San Salvador de Jujuy. Height, weight, bicipital and tricipital skinfolds were measured. Body Mass Index (BMI) and BF\% by BIA (Maltron BF-900) and anthropometry (Deurenberg and Slaugther equations) were determined. Mean and standard deviations were calculated, and the differences by sex and age were established by analysis of variance. Pearson correlation coefficients were calculated, among

El exceso de adiposidad representa en la actualidad uno de los principales problemas de salud pública que afecta a todos los grupos de edad y a ambos sexos, independientemente del origen étnico, cultural o socioeconómico. En niños y adolescentes, se registra una epidemia mundial creciente de sobrepeso (SP) y obesidad $(\mathrm{OB})$, con variación de la tendencia secular entre países (Wang y Lobstein, 2006). En Argentina, en consulta pediátrica (10 a 19 años), el 5,4\% different methods used to estimate $\mathrm{BF} \%$. The agreement between methods was analyzed with Bland-Altman. Females presented higher values of BMI, bicipital and tricipital skifolds, and higher percentages of overweight and obesity. The highest BF\% was obtained with BIA in girls and with Slaugther in boys. Girls had significantly higher mean values of FMI than boys with all 3 methods. The highest $\mathrm{BF} \%$ correlation was observed between BIA and Deurenberg, followed by Slaugther and Deurenberg. There was no agreement between the 3 methods. Given the accessibility of the $\mathrm{BF} \%$ estimation with the Deurenberg equation, the application of this methodology is proposed in epidemiological studies of obesity. Rev Arg Antrop Biol 20(1), 2018. doi:10.17139/raab.2018.0020.01.05

Financiamiento: Secretaría de Ciencia y Técnica y Estudios Regionales.Universidad Nacional de Jujuy. Proyecto "Perfil antropométrico y altura geográfica en poblaciones infanto-juveniles jujeñas" (período 2016-2019).

*Correspondencia a: Jorge Iván Martinez. INECOA-CONICET. Av. Bolivia 1661. 4600 San Salvador de Jujuy. Argentina. E-mail: jorjom@gmail.com

Recibido 20 Marzo 2017; aceptado 06 Julio 2017

doi:10.17139/raab.2018.0020.01.05 
de los sujetos presentan OB y el $20,8 \%$, sobrepeso (SP) (Kovalskys et al., 2011). En 2007, las prevalencias de OB y SP en escolares argentinos fueron del 2,6\% y $19 \%$ respectivamente (Linetzkya et al., 2011). Entre 1995 y 2000, en San Salvador de Jujuy (provincia de Jujuy), utilizando distintos criterios de evaluación, las prevalencias de OB y SP en escolares fueron superiores al $4 \%$ y al $13 \%$ respectivamente (Bejarano et al., 2005).

Por su fácil determinación y su correlación con la adiposidad, el Índice de Masa Corporal (IMC) es el indicador universalmente utilizado para determinar la prevalencia de SP y OB (Gallagher et al., 2000). Para ello se utilizan tres referencias: IOTF (Cole et al., 2000), CDC (Kuczmarski et al., 2002) y OMS (de Onis et al., 2012). Sin embargo, diversos estudios indicaron que estas referencias proporcionan resultados dispares de la prevalencia de ambos trastornos (Padula y Salceda, 2008; Baya Botti et al., 2010; Shields y Tremblay, 2010; Monasta et al., 2011; Meyer et al., 2013). La utilidad del IMC para estimar adiposidad se cuestiona porque: 1) el IMC no diferencia entre la masa grasa (MG) y masa magra (MM) y constituye un pobre predictor de grasa corporal (Wan et al., 2014); 2) la relación entre el IMC y la grasa corporal es dependiente del género, edad y origen étnico de los niños y adolescentes (Daniels et al., 1997). Por estas razones resulta importante recurrir a la determinación lo más exacta posible del porcentaje de grasa corporal (\%GC) y de la MM y MG a través del análisis de la composición corporal.

El modelo clásico de dos componentes plantea a la masa o peso corporal humano total como la suma de dos compartimentos, la MM y la MG. La capacidad de evaluar con precisión la composición corporal en niños y adolescentes, particularmente la MM y la MG, constituye en la actualidad una de las estrategias más eficaces para el diagnóstico, prevención y tratamiento de la obesidad en niños y adolescentes. Entre los métodos para medir el \%GC más eficientes, económicamente más factibles y aplicables en estudios epidemiológicos se cuenta con la antropometría utilizando los pliegues cutáneos y la bioimpedancia (BIA).

Cuando las MM y MG, en kilogramos, obtenidas por cualquier método de determinación del \%GC se expresan como porcentajes del peso total del cuerpo o como pesos absolutos, un joven sano y bien alimentado puede tener valores para estos componentes prácticamente iguales a los de un individuo, de la misma edad, con malnutrición proteicoenergética (VanItallie et al., 1990). Para obviar estas dificultades VanItallie et al. (1990) propusieron utilizar dos índices normalizados por la talla, el Índice de Masa Grasa (IMG) y el Índice de Masa Magra (IMM).

El objetivo del presente estudio es comparar distintos métodos de cálculo del \%GC (antropometría y bioimpedancia) en una población infanto-juvenil jujeña y analizar la variación de la MG, MM y de los IMG e IMM.

\section{MATERIAL Y MÉTODOS}

Este estudio transversal fue llevado a cabo en 232 escolares (142 mujeres; 90 varones) de 12 a 17 años de edad de la ciudad de San Salvador de Jujuy (provincia de Jujuy), localizada a $1259 \mathrm{msnm}$. Se trató de un muestreo por conveniencia que incluyó a los escolares, que contaban con el consentimiento informado de sus padres o tutores y no presentaban ninguna enfermedad evidente al momento de la toma de los datos antropométricos.

La talla, el peso y los pliegues tricipital (PT) y subescapular (PS) se relevaron utilizando los procedimientos estándar (Weiner y Lourie, 1981). La talla se registró con un antropómetro de pie con una precisión de $0,1 \mathrm{~cm}$. El peso, con los niños descalzos y con el mínimo de ropas, fue tomado con una balanza portátil con una precisión de $100 \mathrm{~g}$. Los pliegues fueron medidos con un plicómetro de Harpenden en el lado izquierdo del cuerpo y con una precisión de $0,2 \mathrm{~mm}$.

El estado nutricional de cada individuo se determinó mediante el IMC (peso $(\mathrm{Kg}) /$ talla $\left(\mathrm{m}^{2}\right)$ ) con la referencia IOTF (Cole et al., 2000, 2007).

El \%GC por antropometría se determinó de dos maneras:

1) a partir del IMC utilizando las ecuaciones de Deurenberg et al. (1991):

$$
\begin{aligned}
& \text { Mujeres }=(1,51 * \text { edad })-(0,7 * \text { IMC })-(3,6 * 0)+1,4 \\
& \text { Varones }=(1,51 * \text { edad })-(0,7 * \text { IMC })-(3,6 * 1)+1,4
\end{aligned}
$$


2) a partir de los PT y PS usando la ecuación de Slaughter et al. (1988)

$$
\text { Mujeres }=\left(1,33 * \sum \text { PT PS }\right)-0,013 *\left(\sum \text { PT PS }\right)^{2}-2,5
$$

Varones $=\left(1,21 * \sum\right.$ PT PS $)-0,008 *\left(\sum \text { PT PS }\right)^{2}-1,7$

De acuerdo a Sen y Mondal (2013) para el cálculo de MG, MM, IMG e IMM se utilizaron las siguientes fórmulas:

$$
\begin{gathered}
\text { MG }(\mathrm{kg})=(\% \mathrm{GC} / 100) \times \text { Peso }(\mathrm{kg}) \\
\mathrm{MM}(\mathrm{kg})=\mathrm{MG}(\mathrm{kg})-\text { Peso }(\mathrm{Kg}) \\
\mathrm{IMG}\left(\mathrm{kg} / \mathrm{m}^{2}\right)=\mathrm{MG} / \text { Talla }^{2} \\
\mathrm{IMM}\left(\mathrm{Kg} / \mathrm{m}^{2}\right)=\mathrm{MM} / \text { Talla }^{2}
\end{gathered}
$$

Para la determinación del \%GC por BIA se utilizó un equipo Maltron BF-900 tetrapolar (Maltron International Limited, RU). Los electrodos, autoadhesivos y descartables, se colocaron del lado derecho, dos en la muñeca y dos en el pie. El equipo se calibró permanentemente y las mediciones se realizaron de acuerdo a las instrucciones del equipo luego de un ayuno de $2 \mathrm{hs}$, con la vejiga vacía y en posición supina con los brazos separados del cuerpo y sobre una superficie no conductora.

Los datos se analizaron agrupándolos por sexo y en dos grupos etarios: 12 a 14 años y 15 a 17 años. El análisis estadístico descriptivo de las variables se realizó con media y desvío estándar. Las diferencias por sexo y edad se establecieron mediante análisis de la varianza (ANOVA). Se utilizó una ANOVA de dos vías para controlar la influencia de la edad y el sexo en las variables de composición corporal (MG, MM, IMCG e IMCM). Se calcularon los coeficientes de correlación de Pearson entre los distintos métodos utilizados para estimar el \%GC con un IC del $95 \%$. Para analizar la concordancia entre las distintas medidas del \%GC se utilizó la prueba de Bland Altman (Bland y Altman 1986). Estos análisis se realizaron empleando el software IBM SPSS versión 22.

\section{RESULTADOS}

En la Tabla 1 se observa que, independientemente de la edad, los varones fueron más altos que las mujeres pero éstas presentan valores superiores de IMC, PT y PS. Se registraron diferencias intersexuales estadísticamente significativas para el grupo de 12 a 14 años en la talla, IMC, PT y PS $(\mathrm{p}<0,05)$, y en todas las variables, excepto el IMC, en el grupo de 15-17 años $(p<0,05)$. Los pliegues no presentaron diferen- cias estadísticamente significativas entre grupos de edad en ambos sexos.

En la Tabla 2 se presenta el estado nutricional de la población según el IMC y de acuerdo a las referencias de la IOTF (Cole et al., 2000, 2007). Se observa que la categoría normopeso fue la preponderante independientemente del sexo y la edad. Las mujeres presentaron mayores porcentajes de exceso de adiposidad (sobrepeso y obesidad) con valores que duplicaron a los observados en varones en el grupo de 12 a 14 años. El 12,2\% y 21,8\% de los varones y mujeres respectivamente, presentaron exceso de adiposidad. La prevalencia de delgadez fue baja en ambos sexos (Tabla 2).

$\mathrm{Al}$ analizar el \%GC se observa que los valores difirieron según el método de estimación. Independientemente del sexo, la ecuación de Deuremberg registró los porcentajes más bajos mientras que los mayores \%GC se obtuvieron con BIA en mujeres y con la fórmula de Slaugther en varones (Tabla 3). El \%GC aumentó con la edad en ambos sexos con los tres métodos de estimación, pero las diferencias fueron significativas para Deurenberg y BIA únicamente en varones.

La evaluación de la composición corporal a través del IMM e IMG presentó diferencias intersexuales. Las mujeres presentaron valores promedio significativamente más altos del IMG que los varones con los 3 métodos en los dos grupos etarios considerados (Tabla 4). Dentro del mismo sexo el IMG no presenta diferencias estadísticamente significativas entre grupos de edad con ninguno de los métodos, excepto Slaugther en varones. El IMM presenta resultados similares.

Los \%GC y el IMG obtenidos con antropometría y BIA presentaron una correlación positiva estadísticamente significativa entre sí, en ambos sexos, pero los valores fueron más altos en mujeres. En la Tabla 5 se presentan los coeficientes de correlación independientemente del sexo y edad. La correlación más alta del \%CG se observó entre BIA y Deurenberg, seguida por Slaugther y Deurenberg. La variación del \%GCD, \%GC-S y \%GC-BIA explicaron el 38,1\%, $35,2 \%$ y 33,9\% respectivamente de la variación del IMC. La correlación más alta entre el IMC y el IMG se presentó con Slaugther, seguida por Deurenberg y BIA. 
TABLA 1. Media y desvio estándar de las variables antropométricas por sexo y grupos de edad

\begin{tabular}{|c|c|c|c|c|c|c|c|c|c|c|c|c|}
\hline Sexo & $\begin{array}{c}\text { Edad } \\
\text { (años) }\end{array}$ & $\mathrm{N}$ & $\begin{array}{c}\text { Peso } \\
\text { (kg) }\end{array}$ & & $\begin{array}{l}\text { Talla } \\
(\mathrm{m})\end{array}$ & & IMC & & PT (mm) & & PS (mm) & \\
\hline & & & $\mathrm{X}$ & D.E & $X$ & D.E & $X$ & D.E & $\mathrm{X}$ & D.E & $X$ & D.E \\
\hline \multirow{2}{*}{ Varones } & $12-14$ & 40 & 47,75 & 8,00 & 1,56 & 0,09 & 19,43 & 2,42 & 13,15 & 5,06 & 11,00 & 4,87 \\
\hline & $15-17$ & 50 & 57,19 & 9,76 & 1,65 & 0,06 & 20,91 & 3,26 & 13,88 & 6,50 & 11,04 & 4,46 \\
\hline \multirow{2}{*}{ Mujeres } & $12-14$ & 78 & 49,74 & 11,01 & 1,52 & 0,06 & 21,55 & 4,24 & 18,18 & 5,20 & 13,99 & 5,14 \\
\hline & $15-17$ & 64 & 51,48 & 8,93 & 1,54 & 0,06 & 21,72 & 3,71 & 17,50 & 3,97 & 14,59 & 4,20 \\
\hline
\end{tabular}

IMC:Índice de Masa Corporal; PT:pliegue tricipital; PS:pliegue subescapular; X: media. D.E.:desvío estándar.

TABLA 2. Estado nutricional según la referencia IOTF por sexo y grupos de edad

\begin{tabular}{ccccccccc}
\hline & \multicolumn{4}{c}{ Varones } & \multicolumn{5}{c}{ Mujeres } \\
\cline { 2 - 9 } $\begin{array}{c}\text { Categoría de estado } \\
\text { nutricional }\end{array}$ & \multicolumn{1}{c}{$12-14$ años } & \multicolumn{2}{c}{$15-17$ años } & \multicolumn{2}{c}{$12-14$ años } & \multicolumn{1}{c}{$15-17$ años } \\
& $\mathrm{N}$ & $\%$ & $\mathrm{~N}$ & $\%$ & $\mathrm{~N}$ & $\%$ & $\mathrm{~N}$ & $\%$ \\
\hline Delgadez G $\mathrm{G}^{\circ}$ & 0 & 0,0 & 2 & 4,0 & 1 & 1,3 & 0 & 0,0 \\
Delgadez G $\mathrm{G}^{\circ} 1$ & 1 & 2,0 & 1 & 2,0 & 1 & 1,3 & 4 & 6,3 \\
Normopeso & 35 & 87,0 & 40 & 80,0 & 57 & 73,1 & 48 & 75,0 \\
Sobrepeso & 3 & 7,0 & 6 & 12,0 & 13 & 16,7 & 10 & 15,6 \\
Obesidad & 1 & 2,0 & 1 & 2,0 & 5 & 6,5 & 2 & 3,1 \\
Total & 40 & 100 & 50 & 100 & 77 & 100 & 64 & 100 \\
\hline
\end{tabular}

TABLA 3. Porcentaje de grasa corporal por distintos métodos según sexo y grupos de edad

\begin{tabular}{ccccccccc}
\hline \multirow{2}{*}{ Sexo } & \multirow{2}{*}{ Edad (años) } & $\mathrm{N}$ & \multicolumn{2}{c}{ Deurenberg } & \multicolumn{2}{c}{ Slaugther } & \multicolumn{2}{c}{ BIA } \\
& & & $\mathrm{X}$ & $\mathrm{D} . \mathrm{E}$. & $\mathrm{X}$ & $\mathrm{D} . \mathrm{E}$. & $\mathrm{X}$ & D.E. \\
\hline \multirow{2}{*}{ Varones } & $12-14$ & 40 & 17,50 & 3,78 & 22,2 & 6,98 & 12,90 & 6,80 \\
& $15-17$ & 50 & 12,57 & 3,95 & 22,68 & 7.00 & 16,78 & 7,05 \\
& $12-14$ & 78 & 24,16 & 6,47 & 25,61 & 4,49 & 27,56 & 9,98 \\
\multirow{6}{*}{ Mujeres } & $15-17$ & 64 & 24,38 & 4,51 & 26,16 & 3,11 & 28,76 & 7,93 \\
\hline
\end{tabular}

BIA Bioimpedancia. X:media. D.E.:desvío estándar.

En la Figura 1 se presentan los gráficos de Bland-Altman que permiten visualizar la concordancia entre los distintos métodos de cálculo de la masa grasa. El eje de las abscisas corresponde al valor medio de los métodos comparados y el de las ordenadas a la diferencia entre ambos mé- todos para cada sujeto. En ninguna de las comparaciones la diferencia media (línea discontinua) coincide con la diferencia media de cero (línea continua) lo que indica que, independientemente del sexo y la edad, pocas mediciones concordaron entre los 3 métodos de determinación del \%GC. 
TABLA 4. Media y desvío estándar del IMG e IMM por sexo y grupos de edad

\begin{tabular}{|c|c|c|c|c|c|c|c|c|c|c|c|c|c|c|}
\hline \multirow{2}{*}{ Sexo } & \multirow{2}{*}{$\begin{array}{c}\text { Edad } \\
\text { (años) }\end{array}$} & \multirow{2}{*}{$\mathrm{N}$} & \multicolumn{2}{|c|}{ IMG-D } & \multicolumn{2}{|c|}{ IMG-S } & \multicolumn{2}{|c|}{ IMG-BIA } & \multicolumn{2}{|c|}{ IMM-D } & \multicolumn{2}{|c|}{ IMM-S } & \multicolumn{2}{|c|}{ IMM-BIA } \\
\hline & & & $\mathrm{X}$ & D.E. & $\mathrm{X}$ & D.E. & $\mathrm{X}$ & D.E. & $\mathrm{X}$ & D.E. & $\mathrm{X}$ & D.E. & $\mathrm{X}$ & D.E. \\
\hline \multirow{2}{*}{ Varones } & $12-14$ & 40 & 3,49 & 1,30 & 4,43 & 1,92 & 2,85 & 2,14 & 15,94 & 1,15 & 15,00 & 1,26 & 16,47 & 1,42 \\
\hline & $15-17$ & 50 & 2,75 & 1,39 & 4,90 & 2,22 & 3,68 & 2,09 & 18,16 & 1,9 & 16,02 & 1,76 & 17,18 & 1,80 \\
\hline \multirow{2}{*}{ Mujeres } & $12-14$ & 78 & 5,48 & 2,89 & 5,64 & 1,91 & 6,23 & 3,57 & 16,07 & 1,46 & 15,91 & 2,52 & 15,23 & 1,63 \\
\hline & $15-17$ & 64 & 5,46 & 2,22 & 5,76 & 1,59 & 6,39 & 2,79 & 16,26 & 1,56 & 15,96 & 2,23 & 15,27 & 1,75 \\
\hline
\end{tabular}

IMG-D:Índice de Masa Grasa con fórmula de Deurenberg; IMG-S:Índice de Masa Grasa con fórmula de Slaugther; IMGBIA:Índice de Masa Grasa con Bioimpedancia; IMM-D:Índice de Masa Magra con fórmula de Deurenberg; IMM-S: Índice de Masa Magra con fórmula de Slaugther; IMM-BIA:Índice de Masa Magra con Bioimpedancia; X:media; D.E.: Desvío estándar.

TABLA 5. Correlaciones del porcentaje grasa corporal y del índice masa grasa según métodos con el IMC

\begin{tabular}{cccccccc}
\hline & IMC & $\% G C-D$ & $\% G C-S$ & $\% G C-B I A$ & IMG-D & IMG-S & IMG-BIA \\
\hline IMC & 1 &, $786^{* *}$ &, $625^{* *}$ &, $683^{* *}$ &, $911^{* *}$ &, $895^{* *}$ &, $865^{* *}$ \\
$\%$ GC-D & & 1 &, $605^{* *}$ &, $788^{* *}$ &, $948^{* *}$ &, $762^{* *}$ &, $855^{* *}$ \\
$\%$ GC-S & & & 1 &, $543^{* *}$ &, $585^{* *}$ &, $899^{* *}$ &, $593^{* *}$ \\
$\%$ GC-BIA & & & & 1 &, $760^{* *}$ &, $667^{* *}$ &, $927^{* *}$ \\
IMG-D & & & & & 1 &, $828^{* *}$ &, $907^{* *}$ \\
IMG-S & & & & & & 1 &, $806^{* *}$ \\
IMG-BIA & & & & & & & 1 \\
\hline
\end{tabular}

** $<<0.01$ la correlación es significativa en el nivel 0,01; IMC:Índice de Masa Corporal; \%GC-D:Porcentaje de grasa coporal con fórmula de Deurenberg; \%GC-S:Porcentaje de grasa corporal con fórmula de Slaugther; \%GC-BIA:Porcentaje de grasa corporal con Bioimpedancia; IMG-D:Índice de Masa Grasa con fórmula de Deurenberg; IMG-S:Índice de Masa Grasa con fórmula de Slaugther; IMG-BIA:Índice de Masa Grasa con Bioimpedancia.

\section{DISCUSIÓN}

Este trabajo constituye el primer antecedente sobre la comparación de métodos para evaluar la composición corporal en población juvenil jujeña recurriendo al modelo de dos componentes y empleando bioimpedancia y pliegues cutáneos. Aunque se trata de una muestra pequeña y por conveniencia, los resultados constituyen un aporte a la investigación de la obesidad en poblaciones infanto-juveniles locales. La mayoría de la información sobre la prevalencia de obesidad y sobrepeso en población de esta edad está basada en los puntos de corte proporcionados por el IMC, mientras que la basada en el $\%$ GC por antropometría (pliegues subcutáneos) y bioimpedancia es mucho menos frecuente y casi inexistente para poblaciones argentinas (Rodríguez et al., 2008). El valor de esta información se destaca por la limitación del IMC para distinguir entre MG y MM como ya se comentó previamente.

En la población analizada en general y utilizando métodos relativamente sencillos para la determinación de la masa grasa, se observa una correlación positiva de moderada a alta según los distintos métodos y una concordancia baja entre los mismos. Esto indicaría que las diferentes estimaciones no son intercambiables entre sí, al menos en contextos clínicos, pero que podrían emplearse indistintamente a nivel poblacional en donde interesa analizar la variabilidad 

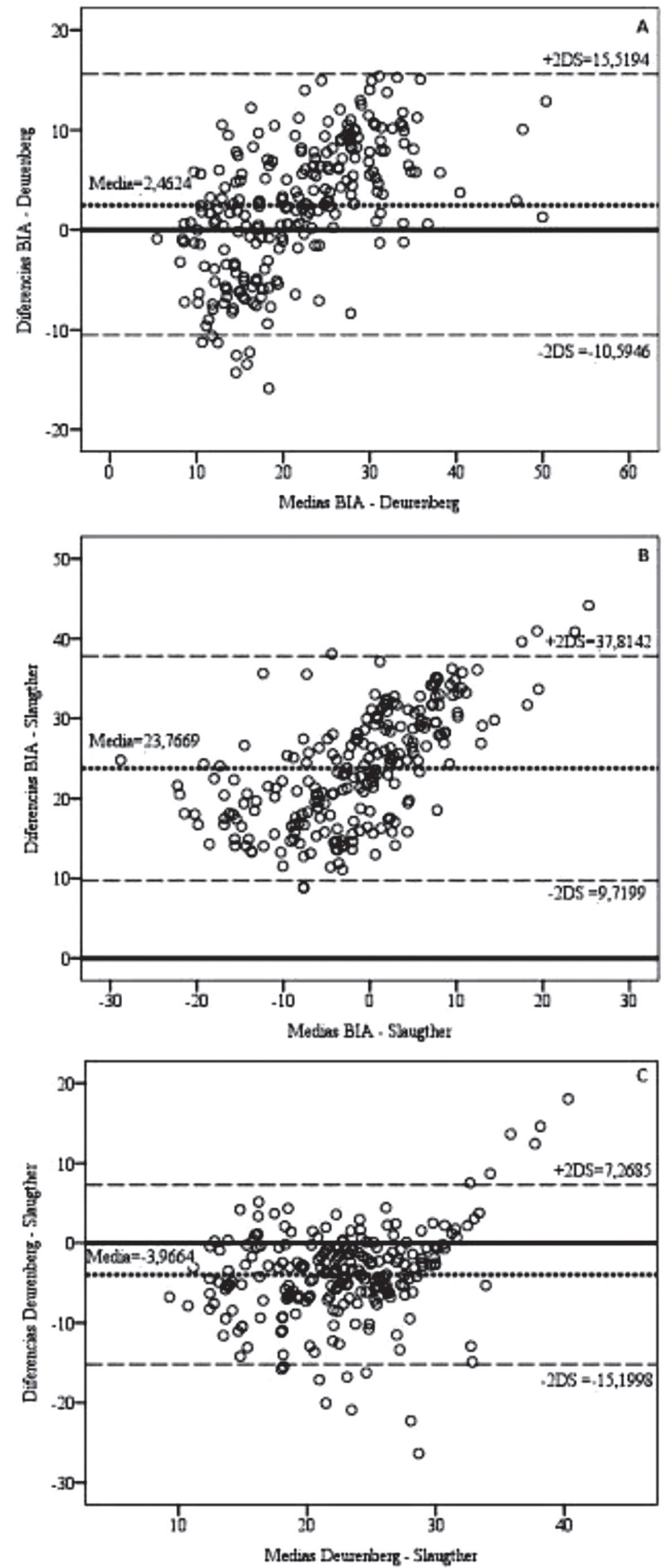

Fig. 1. Prueba de Bland-Altman entre los métodos antropométricos y por bioimpedancia. A (BIA-Deurenberg), B (BIA-Slaugther), C (Deurenberg-Slaugther). 
de la masa grasa por sexo y edad ante diferentes ambientes y no diagnosticar la adiposidad de un individuo en particular. Los resultados encontrados coinciden con otros antecedentes (Eisenmann et al., 2004), particularmente con los de una población infantil argentina de 4-6 años donde el \%GC por BIA se estableció con un equipo semejante al utilizado en este trabajo (Maltron BF-900) (Rodríguez et al., 2008).

El análisis por BIA es un método relativamente simple y económico para estimar el \%GC en estudios epidemiológicos. Existen distintas alternativas en cuanto a los equipos y las ecuaciones provistas por los mismos. Sin embargo, no todos los equipos han sido convenientemente validados y menos aún en población infanto-juvenil. La absorciometría dual de rayos $\mathrm{X}$ (DEXA) es considerado el método de referencia para evaluar la masa grasa y el mismo muestra, según Rodríguez et al. (2008) una correlación moderada $(\mathrm{r}=0,7401, \mathrm{p}<0,001)$ con el \%GC estimado con un equipo Maltron BF-900 en población argentina. En la población jujeña, los \%GC calculados con los distintos métodos varían más en varones que en mujeres (Tabla 3), atribuyéndose esta variación, probablemente, al dimorfismo sexual en la composición corporal propia del crecimiento puberal.

La correlación más alta entre el \%GC estimado con BIA se presenta con el calculado a partir de la ecuación de Deurenberg, independientemente de la edad. Sin embargo, resulta necesario señalar que las ecuaciones de Slaugther et al., (1988) y de Deurenberg et al., (1991) fueron establecidas en poblaciones con un background genético diferente al de las poblaciones jujeñas, con un menor tamaño muestral en el caso de Slaughter y preceden ambas al inicio de la epidemia de obesidad. Lo que podría explicar la falta de concordancia entre los distintos métodos sería la cuestión étnica, debido a que existen evidencias de que la relación entre el \%GC y el IMC exhibe gran heterogeneidad atribuible a diferencias en el balance energético, estructura y densidad corporal libre de grasa, distribución de grasa subcutánea y longitud del segmento inferior en relación con el tamaño del tronco (Deurenberg et al., 1998).

Independientemente de las diferencias étnicas, la longitud de los miembros inferiores, tiene una gran importancia en el cálculo del IMC porque una persona con miembros inferiores relativamente cortos pude tener un IMC mayor en comparación con una persona de la misma talla con miembros inferiores más largos. Esto se debe a que el peso/cm es mayor en el tronco (al que se suma el peso de la cabeza y de los miembros superiores) que el de las piernas (Lomaglio et al., .2009). La relación de la longitud de los miembros inferiores con el IMC tiene particular importancia en las poblaciones infanto-juveniles jujeñas de altura (>2000 msnm) porque estas presentan disminución de la talla, de la talla sentado y de la longitud del miembro inferior (Lomaglio et al., 2009). La estandarización del $\%$ GC por el cuadrado de la talla facilita la interpretación clínica y epidemiológica de las variaciones de la MG y MM en individuos de distinta tallas (VanItallie et al., 1990).

Existe gran dificultad para normalizar el peso y el exceso de grasa en función del tamaño, sexo y edad y este problema es más notorio en la población infanto-juvenil debido a que el crecimiento y los cambios en la composición corporal experimentados durante esta fase de la ontogenia constituyen un factor de confusión (Wells, 2000). Con los tres métodos de determinación del \%GC utilizados en este estudio se observaron diferencias por edad y sexo que se manifiestaron por el hecho de que las mujeres jóvenes presentaron mayor \%GC que los varones de igual edad (Tabla 3). Al analizar la relación del IMC con los IMG calculados con los distintos métodos puede observarse que el componente graso representa el 30,8\%, 27\% y $17 \%$ del IMC según IMG-BIA, IMG-S y IMG$\mathrm{D}$, respectivamente.

De acuerdo a Federico et al. (2011) la asociación entre IMC y \%GC es no lineal entre los varones de 6-12 años de edad y es más fuerte entre los sujetos con sobrepeso y obesidad que en los sujetos con peso normal o bajo. Al correlacionar los IMG calculados con los distintos métodos se observaron, independientemente de la edad y el sexo, valores positivos altos y estadísticamente significativos entre el IMC y el IMG (Tabla 5).

Un método confiable para estimar la composición corporal debe ser seguro, preciso, accesible y aceptable (Aguirre et al., 2015). De los 3 métodos utilizados en este trabajo, el más accesible es el de Deurenberg et al. (1991), ya que 
para aplicarlo solo se necesita conocer el IMC. Esta circunstancia permite su utilización en estudios epidemiológicos y para conocer sólo con la aplicación de una ecuación sencilla el \%GC y el IMG en sujetos con sobrepeso y obesidad.

\section{LITERATURA CITADA}

Aguirre CA, Salazar GDC, Lopez de Romaña DV, Kain J.A, Corvalán CL, Uauy RE. 2015. Evaluation of simple body composition methods: assessment of validity in prepubertal Chilean children. Eur J Clin Nutr 69:269273. doi:10.1038/ejen.2014.144

Baya Botti A, Pérez-Cueto FJA, Vasquez Monllor PA, Kolsteren PW. 2010. International BMI-for-age references underestimate thinness and overestimate overweight and obesity in Bolivian adolescents. Nutr Hosp 25:428-436.

Bejarano IF, Dipierri J, Alfaro E, Quispe Y, Cabrera G. 2005. Evolución de la prevalencia de sobrepeso, obesidad y desnutrición en escolares de San Salvador de Jujuy. Arch Argent Pediatr 103(2):101-109.

Bland JM., Altman DG. 1986. Statistical methods for assessing agreement between two methods of clinical measurement. Lancet 1(8476):307-310. doi:10.1016/ S0140-6736(86)90837-8

Cole TJ, Bellizzi MC, Flegal KM, Dietz WH. 2000. Establishing a standard definition for child overweight and obesity worldwide: international survey. BMJ 320:1240-1243. doi:10.1136/bmj.39238.399444.55

Cole TJ, Flegal KM, Nicholls D, Jackson AA. 2007. Body mass index cut offs to define thinness in children and adolescents: international Survey. BMJ 335(7612):194. doi:10.1136/bmj.39238.399444.55

Daniels SR, Khoury PR, Morrison JA. 1997. The utility of body mass index as a measure of body fatness in children and adolescents: differences by race and gender. Pediatrics 99:804-807. doi:10.1542/peds.99.6.804

Deurenberg P, Weststrate JA, Seidell JC. 1991. Body mass index as a measure of body fatness: age- and sexspecific prediction formulas. Br J Nutr 65:105-114. doi:10.1079/BJN19910073

Deurenberg P, Yap M., van Staveren WA. 1998. Body mass index and percent body fat: a meta analysis among different ethnic groups. Int J Obes Relat Metab Disord 22:1164-1171. doi:10.1038/sj.ijo.0800741

Eisenmann JC, Heelan KA, Welk GJ. 2004. Assessing body composition among 3- to 8-year-old children: anthropometry, BIA, and DXA. Obes Res 12:1633-1640. doi:10.1038/oby.2004.203

Federico B, D〉Aliesio F, Pane F, Capelli G, Rodio A. 2011. Body mass index has a curvilinear relationship with the percentage of body fat among children. BMC Research Notes 4(1):301. doi:10.1186/1756-0500-4-301

Gallagher D, Heymsfield SB, Heo M, Jebb SA, Murgatroyd PR, Sakamoto Y. 2000. Healthy percentage body fat ranges: an approach for developing guidelines based on body mass index. Am J Clin Nutr 72:694-701.

Kovalskys I, Rausch Herscovici C, De Gregorio MJ. 2011. Nutritional status of school-aged children of Buenos Aires, Argentina: data using three references. J Public Health 33:403-411. doi:10.1093/pubmed/fdq079

Kuczmarski RJ, Ogden CL, Guo SS, Grummer-Strawn LM, Flegal KM, Mei Z, Wei R, Curtin LR, Roche AF, Johnson CL. 2002. 2000 CDC Growth Charts for the
United States: methods and development. Vital Health Stat 11:1-190.

Lomaglio D, Dip N, Verón J, Dipierri J, Alfaro E, Bejarano I, Kriscautzky N, Marrodán Serrano M, Mesa Santurino M, Moreno Romero S. 2009. Segmentos corporales de la estatura en niños y adolescentes de poblaciones de altura del noroeste argentino. En: Actas Novenas Jornadas Nacionales de Antropología Biológica. Puerto Madryn: Asociación de Antropología Biológica Argentina. p 37. Disponible en: http://www.fcnym.unlp.edu. ar/aabra/Actas2009/Lomaglio\%20et\%20al..pdf

Linetzkya B, Morello P, Virgolinia M, Ferrante D. 2011. Resultados de la primera encuesta nacional de salud escolar. Argentina. Arch Argent Pediatr 109(2):111-116.

Meyer E, Carrillo R, Román EM, Bejarano IF, Alfaro EL, Dipierri JE. 2013. Prevalencia de sobrepeso y obesidad en escolares jujeños de diferente nivel altitudinal según las referencias IOTF, CDC Y OMS. Arch Argent Pediatr 111(6):516-522. doi:10.5546/aap.2013.516

Monasta L, Lobstein T, Cole TJ, Vignerová J, Cattaneo A. 2011. Defining overweight and obesity in pre-school children: IOTF reference or WHO standard? Obes Rev 12:295-300. doi:10.1111/j.1467-789X.2010.00748.x

de Onis M, Onyango A, Borghi E, Siyam A, Blössner M, Lutter C, WHO Multicentre Growth Reference Study Group. 2012. Public Health Nutr 15(9):1603-1610. doi:10.1017/S136898001200105X

Padula G, Salceda SA. 2008. Comparison between references of the overweight and obesity prevalence, through the body mass index, in Argentinean children. Arch Latinoam Nutr 58:330-335.

Rodríguez PN, Bermúdez EF, Rodríguez GS, Spina MA, Zeni SN, Friedman SM, Exeni RA. 2008. Body composition by simple anthropometry, bioimpedance and DXA in preschool children: interrelationships among methods. Arch Argent Pediatr 106:102-109. doi:10.1590/S0325-00752008000200003

Sen J, Mondal N. 2013. Fat mass and fat-free mass as indicators of body composition among Bengalee muslim children. Ann Hum Biol 40:286-293. doi:10.3109/0301 4460.2013.764014

Shields M, Tremblay MS. 2010. Canadian childhood obesity estimates based on WHO, IOTF and CDC cut-points. Int $\mathrm{J}$ Pediatr Obes 5:265-273. doi:10.3109/17477160903268282

Slaughter MH, Lohman TG, Boileau RA, Horswill CA, Stillman RJ, Van Loan MD, Bemben DA. 1988. Skinfold equations for estimation of body fatness in children and youth. Hum Biol 60:709-723.

VanItallie TB, Yang MU, Heymsfield SB, Funk RC, Boileau RA. 1990. Height-normalized indices of the body's fatfree mass and fat mass: potentially useful indicators of nutritional status. Am J Clin Nutr 52:953-959.

Wan CS, Ward LC, Halim J, Gow ML, Ho M, Briody JN, Leung K, Cowell CT, Garnett SP. 2014. Bioelectrical impedance analysis to estimate body composition, and change in adiposity, in overweight and obese adolescents: comparison with dual-energy $\mathrm{x}$-ray absorptiometry. BMC Pediatrics 14:249. doi:10.1186/1471-243114-249

Wang Y, Lobstein T. 2006. Worldwide trends in childhood overweight and obesity. Int J Pediatr Obes 1 (1):11-25. doi:10.1080/17477160600586747

Weiner JS, Lourie JA. 1981. Practical human biology. London: Academic Press.

Wells JC. 2000. A Hattori chart analysis of body mass index in infants and children. Int J Obes Relat Metab Disord 24:325-329. doi:10.1038/sj.ijo.0801132 